'If everyone in the music business were brutally honest about what their intentions were then you could sort things out, but it's all smoke and mirrors.' So said Noel Gallagher of Oasis. In this month's Airwaves, we discuss music, smoke and mirrors and of course respiratory science!

\section{CIGARETTES AND ALCOHOL}

In the early nineties, Oasis famously sang: "... all I need are cigarettes and alcohol..." But will the fags protect the Gallaghers against death from COVID-19? In the early stages of the pandemic some epidemiological studies concluded that smoking was associated with lower risks of COVID-19 mortality. In this month's Thorax (see page 65) Clift and colleagues explode this myth with a Mendelian randomisation study. The study cohort comprises almost 300000 white British participants from the UK Biobank, with data on smoking exposure. In addition, the researchers identified genetic proxies for smoking initiation and smoking heaviness. The team used record linkage to collate outcome data from COVID-19 testing results, hospital admissions, and death certificates. The authors report a positive association between smoking and risk of COVID-19-related death. Genetically predicted smoking heaviness was strongly associated with death from COVID-19. The authors suggest that the "table 2 fallacy" may explain the previous studies which suggested that smoking protects. In a linked editorial (see page 7), Laverty and Millet suggest that the pandemic should focus our minds on tobacco control and point out that tobacco kills more people per year than have died as a result of COVID-19. The Triumvirate agree that tobacco control should be our top priority. As Oasis put it: "You gotta make it happen".

\section{CYSTIC, SICKLE CELL AND REM}

"I will try not to breathe, This decision is mine, I have lived a full life". This song, on REM's album "Automatic for the People" is thought to be have been written when one of the band members fell ill. However, the 22 patients with sickle cell disease who experienced difficulty breathing in the brief communication from Kort and

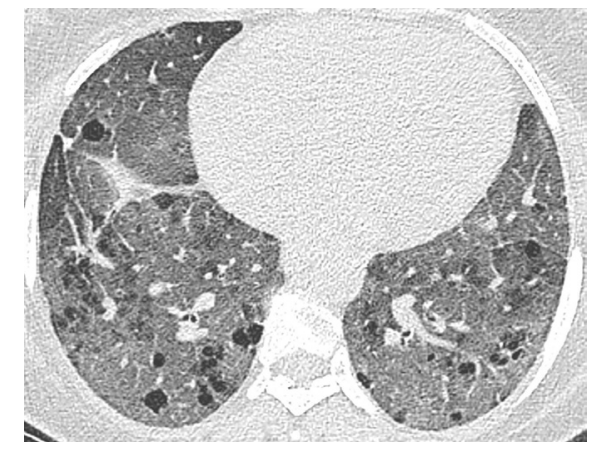

colleagues (see page 91) had not yet lived a full life (mean age 37 years). The authors describe diffuse cystic lung disease (see figure) in sickle cell disease and conduct a case control study to explore the mechanism. In the index case, the bronchioles showed constrictive bronchiolitis. In the case control study, pulmonary function testing showed significantly lower forced expiratory flow $25 \%$ to $75 \%$ of vital capacity) in cases vs controls. The authors speculate that this indicates a bronchiolar mechanism underlying the diffuse cystic lung disease. A bronchiolar mechanism was clearly what REM had in mind when they sang: "I shudder to breathe."

\section{THE FINAL COUNTDOWN}

As the Triumvirate enters the final countdown I am reminded we are leaving together. We may not be headed to Venus, but we stand tall. Another insalubrious habit to leave is smoking, and in this issue of Thorax, Siddiqi and colleagues (see page 74) describe the double blind randomised control trial of cystine to promote smoking cessation in 2472 patients with pulmonary TB. $25 \%$ of patients managed to quit smoking and those who quit had better TB cure plus treatment completion rates and lower TB relapse rates as well as lower TB clinical scores. So patients who quit smoking have generally better outcomes from $\mathrm{TB}$, and therefore in contrast with the classic song from "Europe", patients with TB should not miss smoking so, "... with so many light years to go".

\section{I'LL FEEL A WHOLE LOT BETTER}

Chronic hypersensitivity pneumonitis $(\mathrm{CHP})$ is a progressive fibrotic lung disease that occurs following exposure to a range of well described organic antigens. It is therefore not surprising that The Byrds should write a song about antigen removal on their album $\mathrm{Mr}$ Tambourine Man. However, as they sing "And I'll probably feel a whole lot better when you're gone" they implicitly acknowledge that antigen removal is not a guarantee and that there are other determinants of prognosis. In this issue of Thorax (see page 86) Fernandez Perez and colleagues use RNA sequencing in a small cohort of patients with stable vs progressive CHP describe the prognostic accuracy of a peripheral blood transcriptome signature comprising 74 differentially expressed transcripts in $\mathrm{CHP}$ and suggest it out performs models based entirely on clinical features such as baseline lung function and radiological fibrosis. So perhaps now we know the reasons why, "I have to let you go, and run away, after what you did (to the transcriptome), you can't stay."

\section{SMOKE WITHOUT FIRE}

Primal fear, a German power metal band, told us that 'there's no smoke without fire'. The data from Hashem and colleagues (see page 22) focuses on smouldering, smoke and fire with a particular focus on the hypoinflammatory ('smouldering') and hyperinflammatory ('smoke and fire') subphenotypes observed in acute respiratory distress syndrome (ARDS). The investigators report 6 month and 12 month patient outcomes based on inflammatory subphenotypes in sepsis-associated ARDS, as part of the secondary analysis of SAILSALTOS trial. 568 patients were included in the study and the relationship between subphenotype status and physical function, cognition, mental health symptoms and quality of life investigated. There was no significant difference in survival beyond 90 days and no differences in 6 month or 12 month physical, cognitive and mental health outcomes between the subphenotypes. The separation between smouldering hypoinflammation and smoke and fire the hyperinflammatory subphenotypes of ARDS has no long-term impact.

(C) Author(s) (or their employer(s)) 2022. No commercial re-use. See rights and permissions. Published by BMJ. 